\title{
Fe K line profile in PG quasars: the averaged shape and Eddington ratio dependence
}

\author{
H. Inoue,${ }^{1}$ Y. Terashima,${ }^{2}$ and L. C. $\mathrm{Ho}^{3}$ \\ ${ }^{1}$ Institute of Space and Astronautical Science, 3-1-1 Yoshinodai, Sagamihara 229-8510, Japan \\ ${ }^{2}$ Ehime Univesity, 2-5 Bunkyo, Matsuyama 790-8577, Japan. \\ ${ }^{3}$ The Observatories of the Carnegie Institution of Washington, 813 Santa Barbara Street, \\ Pasadena, CA 91101-1292
}

\begin{abstract}
Fe $\mathrm{K}$ emission line is a powerful probe of the inner part of an accretion disk. We analyze X-ray spectra of 43 Palomar-Green (PG) quasars taken from Boroson \& Green (1992) observed with $X M M$-Newton and make an averaged Fe K line profile. We study the Eddington ratio dependence of the Fe $\mathrm{K}$ line profile. The width of the Fe line becomes broader $(\sigma=0.1$ to $0.7 \mathrm{keV}$ ) and its peak energy becomes higher (6.4 to $6.8 \mathrm{keV}$ ) as the Eddington ratio gets larger.

These results indicate that the physical state of the accretion disk, such as the geometrical structure and/or ionization state, changes with the Eddington ratio.
\end{abstract}

Keywords. Quasars - X-rays - accretion, accretion disks

\section{The sample}

We selected 43 PG quasars (Boroson \& Green 1992) observed with EPIC-PN onboard $X M M-N e w t o n$. Their redshifts, central black hole masses, and Eddington ratios are well known from optical spectra. The quasars in our sample have relatively low redshifts $(z \leqslant 0.5)$, and the central black hole masses range widely from $10^{6}$ to $10^{9} M_{\odot}$. The Eddington ratio, which is one of the most important parameters to characterize an accretion disk, ranges from 0.05 to 4.50 . This wide range enables us to study the Eddington ratio dependence of the Fe line profile.

\section{Results}

We fitted the spectra of the 43 quasars systematically by using a canonical model consisting of a power-law, a blackbody, an edge for objects showing signature of warm absorbers, and a Gaussian, all modified by the Galactic absorption. The spectra of 38 objects are well reproduced by this model. The spectra of the remaining five objects require an additional absorbed power law with a column density of $10^{23} \mathrm{~cm}^{-2}$.

In order to characterize the mean Fe K line profile quantitatively, we fitted all spectra simultaneously with a model consisting of the best-fit continuum model and a Gaussian. The Fe K line in each quasar has a different peak energy in the observed frame according to their redshifts. In order to properly treat the detector response, which is energy dependent, we applied simultaneous fits rather than fits to a composite spectrum. A common peak energy and a width were used for the Gaussian and the normalizations were left free individually. The best-fit parameters are $E=6.48_{-0.04}^{+0.05} \mathrm{keV}, \sigma=0.36_{-0.08}^{+0.08} \mathrm{keV}$, and $\mathrm{EW}=248 \pm 168 \mathrm{eV}$, where the $\mathrm{EW}$ is the mean equivalent width and its error is a standard deviation of the distribution of the EW.

The sample was then divided into four groups according to the Eddington ratio and averaged spectra were derived in each group. The co-added spectra are shown in Figure 1 

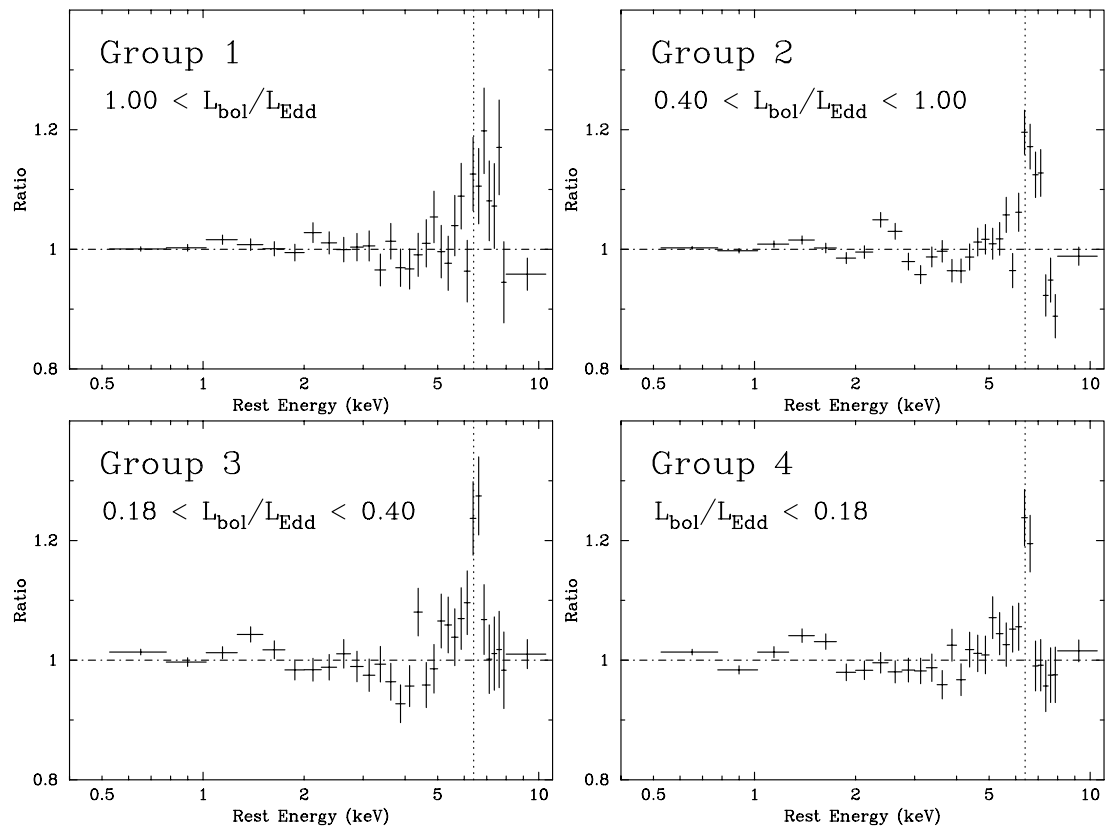

Figure 1. Eddington ratio dependence of the Fe-K line profile. The vertical dotted line is at the energy $6.4 \mathrm{keV}$.
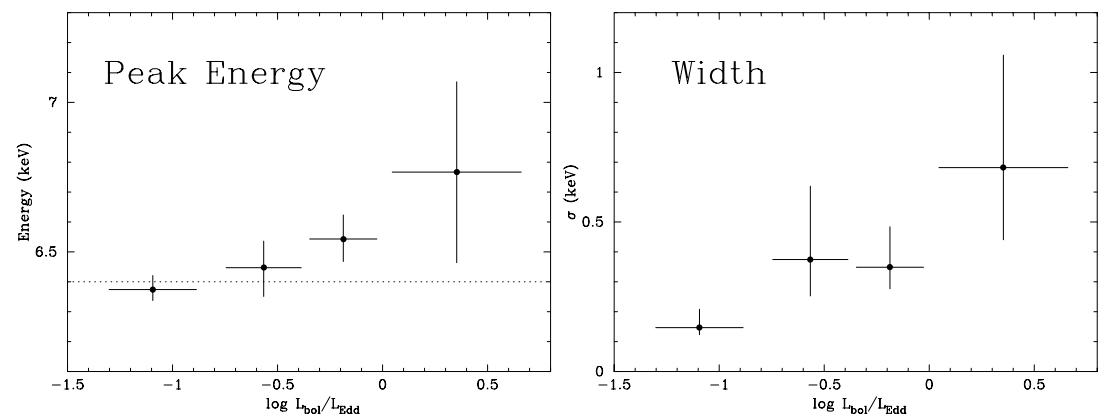

Figure 2. Eddington ratio dependence of the peak energy (left) and the width (right). The horizontal dotted line is at the energy $6.4 \mathrm{keV}$.

for a presentation purpose. We fitted the spectra in each group simultaneously to quantify the Fe line shape. The best-fit parameters are shown in Figure 2. The peak energy of the Fe $\mathrm{K}$ line becomes higher $(6.4$ to $6.8 \mathrm{keV})$ and its width becomes broader $(\sigma=0.1$ to $0.7 \mathrm{keV}$ ) as the Eddington ratio gets larger. The mean equivalent width is $\mathrm{EW} \approx 280 \mathrm{eV}$ except for the lowest Eddington ratio group $(\mathrm{EW} \approx 130 \mathrm{eV})$. These results indicate that the physical state of the accretion disk, such as the inner radius of the optically thick disk and/or ionization state, changes with the Eddington ratio.

\section{References}

Boroson, T. A. \& Green, R. F. 1992, ApJS, 80, 109

Jiménez-Bailón, E. et al. 2005, A\&A, 435, 449

Page, K. L. et al. 2004, MNRAS, 347, 316

Piconcelli, E. et al. 2005, A\&A, 432, 15

Streblyanska, A. et al. 2005, A\&A, 432, 395

Tanaka, Y. et al. 1995, Nature, 375, 659 